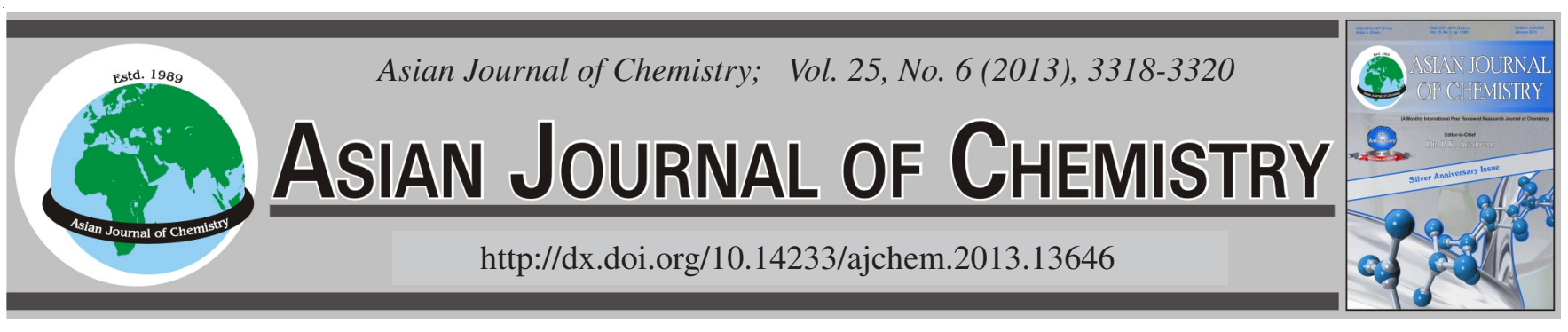

\title{
Emulsion Properties of Poly(n-butyl acrylate)/Poly(methyl methacrylate) Polymer with Core-Shell Structure
}

\author{
Guo-Quan Zhu, Fa-Gang Wang ${ }^{*}$, Ke-Jing Xu and Yu-Ying Liu
}

School of Materials Science and Engineering, Shandong University of Technology, Zibo 255049, P.R. China

*Corresponding author: E-mail: fagangwang@126.com

\begin{abstract}
Poly( $n$-butyl acrylate)/poly(methyl methacrylate) core-shell structure polymer emulsion was synthesized by two-stage emulsion polymerization with sodium dodecyl sulfonate and polyoxyethylene nonylphenol ether (OP-10) as composite emulsifier, distilled water as the continuous medium and potassium persulfate as the initiator. Differential scanning calorimetry and dynamic light scattering were used to investigate the compatibility of two polymers and the size of Poly(n-butyl acrylate)/poly(methyl methacrylate) core-shell polymer particles, respectively.
\end{abstract}

Key Words: Poly( $n$-butyl acrylate), Poly(methyl methacrylate), Seed emulsion polymerization, Core-shell.

\section{INTRODUCTION}

It is well known that core-shell polymers are attracting scientific and industrial interests because their chemical and mechanical properties can be tailored for applications such as paints, adhesives, paper and textile manufacturing, reinforced elastomers, high-impact plastics and toughened plastics ${ }^{1-9}$. Usually, core-shell polymers are made by two-stage emulsion polymerization; first one monomer is polymerized to form the core, then the second monomer is added in batch or semicontinuously and polymerized over the seeded particles to form the shell ${ }^{10,11}$.

Emulsion polymerization is an important and widely used process for the manufacture of polymer products ${ }^{12-14}$. It involves the propagation reaction of free radicals with monomer within the monomer-swollen polymer particles dispersed in the continuous aqueous phase ${ }^{13}$. The discrete hydrophobic particles are stabilized by surfactant such as the anionic sodium dodecyl sulfate. Micelles are formed when the level of surfactant is greater than its critical micelle concentration. Particle nuclei are generated via the capture of radicals by micelles, termed the micelle nucleation. The water-borne radical becomes insoluble and forms a particle nucleus when a critical chain length is achieved. This is followed by the formation of stable primary particles via the limited flocculation of unstable particle nuclei and adsorption of surfactant on their particle surfaces ${ }^{13,15}$.

In this paper, poly(n-butyl acrylate)/poly(methyl methacrylate) core-shell polymer emulsion was synthesized by two- stage emulsion polymerization. Differential scanning calorimetry and dynamic light scattering were used to investigate the properties of poly( $n$-butyl acrylate)/poly(methyl methacrylate) core-shell polymer. Some properties of poly(n-butyl acrylate)/ poly(methyl methacrylate) random copolymers were also involved. Poly( $n$-butyl acrylate)/poly(methyl methacrylate) random copolymers were synthesized by traditional emulsion polymerization.

\section{EXPERIMENTAL}

Methyl methacrylate and $n$-butyl acrylate were washed several times first with a $10 \mathrm{wt} \%$ aqueous solution of sodium hydroxide and afterwards with distilled-and-deionized water until wash waters were neutral. Then, they were dried over $\mathrm{CaCl}_{2}$ and distilled under reduce pressure and stored at $8-10^{\circ} \mathrm{C}$ until use. Potassium persulfate and all other chemicals were of analytical grade and used without further purification.

Preparation of poly(n-butyl acrylate)/poly(methyl methacrylate) core-shell polymer: Poly(n-butyl acrylate) seed latex was prepared by emulsion polymerization at $70^{\circ} \mathrm{C}$, using butyl acrylate as monomer, sodium dodecyl sulfonate and polyoxyethylene nonylphenol ether as composite emulsifier, potassium persulfate as initiator and distilled water as solvent. Briefly, a $500 \mathrm{~mL}$ round-bottomed, four-necked flask was fitted with a mechanical stirrer, thermometer, condenser and a dropping funnel. Reaction was carried out at a constant temperature in a water bath under a nitrogen atmosphere. At $40^{\circ} \mathrm{C}$, monomer, composite emulsifier and distilled water were put into the reactor and pre-emulsified with the stirring speed 
of $200 \mathrm{rpm}$ for $35 \mathrm{~min}$. When the temperature of emulsion achieved $70{ }^{\circ} \mathrm{C}$, appropriate amount of initiator was put into the reactor to initiate reaction. The emulsion polymerization was maintained for $2 \mathrm{~h}$.

When the temperature of poly( $n$-butyl acrylate) emulsion dropped to $40^{\circ} \mathrm{C}$, methyl methacrylate were put into the reactor and stirred adequately for $35 \mathrm{~min}$. Then, the temperature of emulsion was increased. When the temperature of emulsion reached $70{ }^{\circ} \mathrm{C}$, appropriate amount of initiator was added into the reactor. Differently, no additional emulsifier was added. The emulsion polymerization was retained $1.5 \mathrm{~h}$. Usually, as a little of butyl acrylate monomers un-reacted existed in the first-stage emulsion polymerization, a small quantity of poly(BA-co-MMA) random copolymers as transition-zone formed in the second-stage emulsion polymerization.

Preparation of $\operatorname{poly}(\boldsymbol{n}$-butyl acrylate)/poly(methyl methacrylate) core-shell polymer membrane: Thin core-shell polymer membrane was prepared by casting the polymer emulsion onto the clean surface of glass, then the membrane was dried at $50{ }^{\circ} \mathrm{C}$ under vacuum.

Test methods: Differential scanning calorimetry measurements over the temperature range from 200 to $450 \mathrm{~K}$ were made on a differential scanning calorimetry Q100 (TA, USA) at a heating rate of $20{ }^{\circ} \mathrm{C} / \mathrm{min}$ under a nitrogen purge. The weight of each sample was $10 \mathrm{mg}$.

Dynamic light scattering measurements were made using a S4700 (Malvern Instrument, UK) with an argon laser beam at a wavelength of $488 \mathrm{~nm}$ at $25^{\circ} \mathrm{C}$. A scattering angle of $90{ }^{\circ} \mathrm{C}$ was used.

\section{RESULTS AND DISCUSSION}

Differential scanning calorimetry measurements: Table-1 shows the differential scanning calorimetry data of poly(BA-co-MMA) random copolymer and poly(n-butyl acrylate)/poly(methyl methacrylate) core-shell polymer, where the mole ratio of butyl acrylate to methyl methacrylate is $1: 1$. As it can be seen from Table-1, poly(BA-co-MMA) random copolymer and poly(n-butyl acrylate)/poly(methyl methacrylate) core-shell polymer have one glass transition temperature $\left(\mathrm{T}_{\mathrm{g}}\right)$ and three glass transition temperature, respectively. For poly(BA-co-MMA) random copolymer, the only $\mathrm{T}_{\mathrm{g}}$ (at 286 $\mathrm{K})$ could be attributed to the same component existing in random copolymer. For poly( $n$-butyl acrylate)/poly(methyl methacrylate) core-shell polymer, the low- $\mathrm{T}_{\mathrm{g}}$ (at $226 \mathrm{~K}$ ), the middle- $\mathrm{T}_{\mathrm{g}}$ (at $289 \mathrm{~K}$ ) and the high- $\mathrm{T}_{\mathrm{g}}$ (at $372 \mathrm{~K}$ ) correspond to poly(n-butyl acrylate) component, poly(BA-co-MMA) component and poly(methyl methacrylate) component, respectively. Comparing with $\mathrm{T}_{\mathrm{g}}$ data of poly ( $n$-butyl acrylate) homopolymer $(219 \mathrm{~K})$ and poly(methyl methacrylate) homopolymer (377 $\mathrm{K})$, it is found that the $\mathrm{T}_{\mathrm{g}}$ of poly ( $n$-butyl acrylate) component in poly (n-butyl acrylate)/poly(methyl methacrylate) core-shell polymer is higher than that of poly( $n$-butyl acrylate) homopolymer and the $\mathrm{T}_{\mathrm{g}}$ of poly(methyl methacrylate) component is lower than that of poly(methyl methacrylate) homopolymer. As known, the existence of a little of poly(BA-co-MMA) random copolymer as transition-zone in poly(n-butyl acrylate)/ poly(methyl methacrylate) core-shell polymer promotes the miscibility and the interaction between poly(n-butyl acrylate) and poly(methyl methacrylate) component. That is to say, the shift of $\mathrm{T}_{\mathrm{g}}$ can be attributed to the existence of a small quantity of poly(BA-co-MMA) random copolymer in poly( $n$-butyl acrylate)/poly(methyl methacrylate) core-shell polymer.

TABLE-1
DIFFERENTIAL SCANNING CALORIMETRY DATA OF
P(BA-co-MMA) RANDOM COPOLYMER AND
PBA/PMMA CORE-SHELL POLYMER ${ }^{\mathrm{a}}$

Dynamic light scattering measurments: Table- 2 presents the size of poly( $n$-butyl acrylate) seed particles and $\operatorname{poly}(n-$ butyl acrylate)/poly(methyl methacrylate) core-shell polymer particles, where the emulsifier concentration is $2 \mathrm{wt} . \%$, the weight ratio of deionized water to (butyl acrylate + methyl methacrylate) is $3: 1$, the mole ratio of butyl acrylate to methyl methacrylate is $1: 1$. As seen from Table-2, the average particle diameter of poly(n-butyl acrylate)/poly(methyl methacrylate) core-shell polymer particles is larger than that of poly $(n$-butyl acrylate) seed particles. This phenomenon demonstrated that the formation of methyl methacrylate shell layers outside the poly(n-butyl acrylate) seed particles.

TABLE-2

SIZE OF PBA SEED PARTICLES AND PBA/PMMA CORE-SHELL POLYMER PARTICLES ${ }^{a}$

\begin{tabular}{lc}
\hline Sample & Average particle diameter (nm) \\
\hline PBA seed particles & 65.1 \\
Core-shell polymer particles & 108.4 \\
\hline & athe emulsifier concentration is $2 \mathrm{wt} \%$, the weight ratio of deionized \\
water to (butyl acrylate + methyl methacrylate) is $3: 1$, the mole ratio \\
of butyl acrylate to methyl methacrylate is $1: 1$
\end{tabular}

Fig. 1 indicates the relationship between average particle diameter of core-shell polymer particles and methyl methacrylate content in (butyl acrylate + methyl methacrylate), where the emulsifier concentration is $2 \mathrm{wt} \%$, the weight ratio of deionized water to (butyl acrylate + methyl methacrylate) is 3:1. As is shown in Fig. 1, the average particle diameter of core-shell polymer particles is linearly proportional to methyl methacrylate content. In other words, at the same emulsifier content, the increase of methyl methacrylate content upgrades the average particle diameter of core-shell polymer particles.

Effects of methyl methacrylate content on the minimum membrane formation temperature of core-shell polymer: Fig. 2 shows the effects of methyl methacrylate content on the minimum membrane formation temperature (Mini MFT) of core-shell polymer, where the emulsifier concentration is $2 \mathrm{wt}$ $\%$, the weight ratio of deionized water to (butyl acrylate + methyl methacrylate) is 3:1. As shown in Fig. 2, the Mini MFT of core-shell polymer increases with the augment of methyl methacrylate content. As noted, methyl methacrylate as hard monomer holds lower viscidity and the lower viscidity of methyl methacrylate monomer could affect the formation of core-shell polymer membrane. That is to say, the increase of 


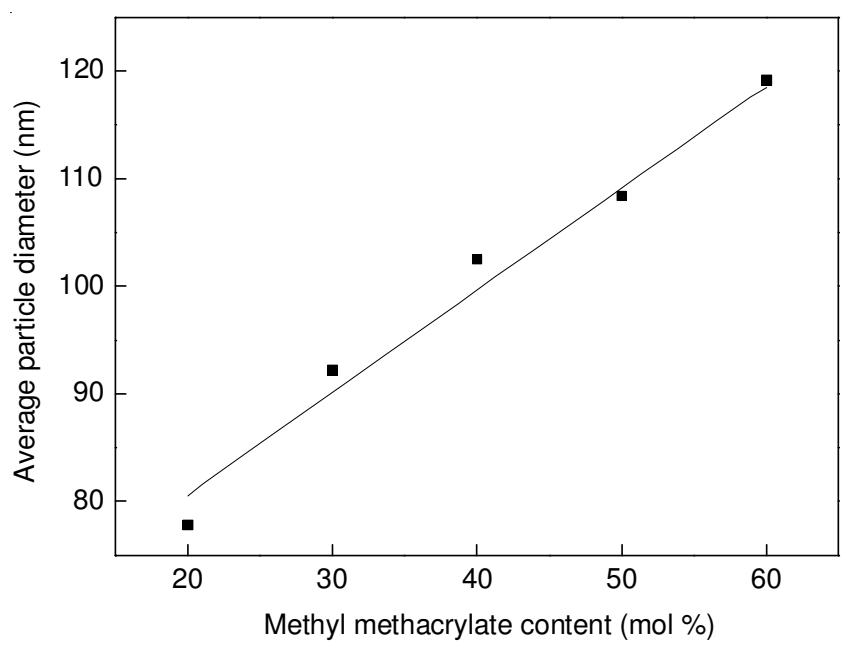

Fig. 1. Relationship between average particle diameter of core-shell polymer particles and methyl methacrylate content in (butyl acrylate + methyl methacrylate), where the emulsifier concentration is $2 \mathrm{wt}$ $\%$, the weight ratio of deionized water to (butyl acrylate + methyl methacrylate) is $3: 1$

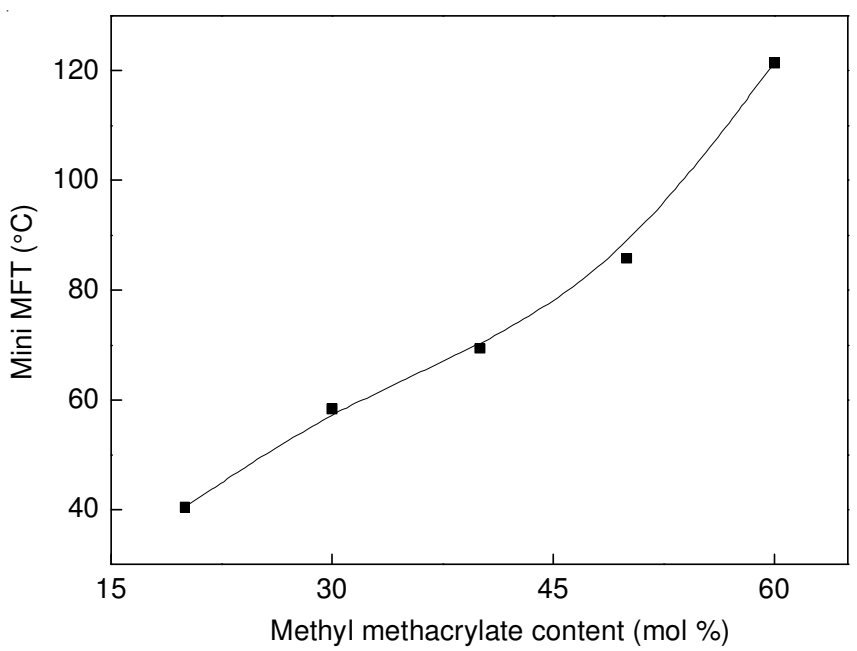

Fig. 2. Effects of methyl methacrylate content on the Mini MFT of coreshell polymer, where the emulsifier concentration is $2 \mathrm{wt} \%$, the weight ratio of deionized water to (butyl acrylate + methyl methacrylate) is $3: 1$

the Mini MFT of core-shell polymer could be attributed to the lower viscidity of methyl methacrylate monomer. In order to make the core-shell polymer membrane holds better hardness and lower Mini MFT, methyl methacrylate content in (butyl acrylate + methyl methacrylate) was usually controlled between (30-50) mol \%.

\section{Conclusion}

Poly(n-butyl acrylate)/poly(methyl methacrylate) coreshell polymer emulsion was synthesized by two-stage emulsion polymerization. Differential scanning calorimetry data verified that core-shell polymers have three glass transition temperatures $\left(\mathrm{T}_{\mathrm{g}} \mathrm{s}\right)$ corresponding to poly ( $n$-butyl acrylate) component, poly(BA-co-MMA) component and poly(methyl methacrylate) component, respectively. Dynamic light scattering results testified that, at the same emulsifier content, the average particle diameter of core-shell polymer particles increases with the augment of methyl methacrylate content. The membrane formation tests demonstrated that the minimum membrane formation temperature of core-shell polymer increases with the augment of methyl methacrylate content.

\section{ACKNOWLEDGEMENTS}

This work is supported by the Natural Science Foundation of Shandong Province (No. ZR2011EMM009).

\section{REFERENCES}

1. A.K. Khan, B.C. Ray and S.K. Dolui, Prog. Org. Coat., 62, 65 (2008).

2. A.M. Santos, A. Elaissari, J.M.G. Martinho and C. Pichot, Polymer, 46, 1181 (2005).

3. C.J. Ferguson, G.T. Russell and R.G. Gilbert, Polymer, 43, 4557 (2002).

4. M. Ishida, J. Oshima, K. Yoshinaga and F. Horii, Polymer, 40, 3323 (1999).

5. H.C. Kim, K.H. Choi and S.K. Park, Asian J. Chem., 24, 4185 (2012).

6. F. Henry, F. Cansell, J.L. Guillamine and C. Pichot, Colloid. Polym. Sci., 267, 167 (1989).

7. G.J. Wang, C.S. Kang and R.G. Jin, Prog. Org. Coat., 50, 55 (2004)

8. C.J. Ferguson, G.T. Russell and R.G. Gilbert, Polymer, 43, 6371 (2002).

9. L. Rios, M. Hidalgo, J.Y. Cavaille, J. Guillot, A. Guyot and C. Pichot, Colloid. Polym. Sci., 269, 812 (1991).

10. M. Hidalgo, J.Y. Cavaille, J. Guillot, A. Guyot, C. Pichot and L. Rios, Colloid. Polym. Sci., 270, 1208 (1992).

11. J.W. Ha, L.J. Park and D.K. Kim, Macromolecules, 35, 6811 (2002).

12. M. Jain, R.A. Vora and U.S. Satpathy, Eur. Polym. J., 39, 2069 (2003).

13. C.S. Chen, J.J. Lin, Y.L. Lin and S.Z. Lai, Eur. Polym. J., 42, 1033 (2006).

14. I.A. Maxwell, B.R. Morrison, D.H. Napper and R.G. Gilbert, Macromolecules, 24, 1629 (1991).

15. Y.S. Choi, M.H. Choi, K.H. Wang, S.O. Kim, Y.K. Kim and I.J. Chung, Macromolecules, 34, 8978 (2001). 